\title{
Seroepidemiology of Chikungunya Fever in Dhaka, Bangladesh: A Cross- sectional Study
}

\author{
Abiral Hasib Shourav ${ }^{1}$, M. Murshida Mahbub ${ }^{2}$, Mahmuda Yasmin ${ }^{1}$, Chowdhury Rafiqul Ahsan ${ }^{1}$ and \\ Jamalun Nessa ${ }^{1 *}$ \\ ${ }^{I}$ Department of Microbiology, University of Dhaka, Dhaka-1000, Bangladesh, ${ }^{2}$ Department of Genetic Engineering and Biotechnology, East West University, A/ \\ 2 Jahurul Islam Avenue, Aftabnagar, Dhaka-1212, Bangladesh
}

\begin{abstract}
Chikungunya fever (CHIKF) is a mosquito-borne febrile illness caused by the Chikungunya virus (CHIKV). Bangladesh has documented several outbreaks of CHIKF since it was first reported in 2008. The latest CHIKF outbreak occurred in the Dhaka in 2017. In this study, a serosurvey of the 2017 outbreak was conducted during its peak. The study involved the assessment of CHIKV immunoreactions among participants suffering from CHIKF related symptoms. One hundred blood samples were collected from patients suffering from CHIKF-associated symptoms and were subsequently tested for the presence of anti-CHIKV IgM and/or IgG antibodies. Data based on clinical symptoms and the demographics of the participants were recorded and analyzed. Seventy-four percent of the studied patients $(n=100)$ possessed anti-CHIKV antibodies. Among this seropositive group $(n=74)$, almost $62 \%$ contained anti-CHIKV IgM and IgG antibodies, whereas $10 \%$ contained only anti-CHIKV IgM antibodies indicating recent infection. Anti-CHIKV IgG antibodies were found in $28 \%$ of patients. Among the symptoms examined, polyarthralgia showed a highly significant relationship $(\mathrm{P}<0.001)$, whereas high fever and the presence of rash demonstrated a significant association with CHIKV seropositivity $(\mathrm{P}<0.05)$. Discoveries made on this research can better help public health officials to gain a comprehensive insight into the seroepidemiology of the condition based in the city and maintain constant vigilance against any future outbreak.
\end{abstract}

Keywords: Chikungunya Virus, Seroepidemiology, Dhaka, Bangladesh.

\section{Introduction}

The Chikungunya virus (CHIKV) is an alphavirus of the Togaviridae family first discovered in 1952-1953 in Tanzania ${ }^{1}$. The virus is transmitted by the female Aedes mosquitoes, mainly the $A$. aegypti and $A$. albopictus ${ }^{1,2}$. The most prominent symptoms of the disease are high fever, polyarthralgia, myalgia, headache and maculopapular rash. Other notable symptoms may include nausea and vomiting. Fever and rash last for a very short time, but the pain in the joint is incapacitating and persistent. CHIKV infection of a population lacking herd immunity usually produces a massive epidemic affecting an enormous number of people. The interactions of some factors bring about this occurrence: vulnerable human populations, availability of the vectors $A$. aegypti and $A$. albopictus and viral mutations that augment its infectivity ${ }^{2}$.

CHIKV infection can be diagnosed based on clinical, epidemiological and laboratory findings ${ }^{3}$. The sudden appearance of high-grade fever and debilitating pain in the joint are the major criteria for clinical diagnosis. However, in parts of the world where Chikungunya, Dengue and Zika viruses co-exist, clinical diagnosis of CHIKF can be difficult ${ }^{4}$. Diagnosis using epidemiological data can be done by the use of the suspected patient's travel history to areas that going through a CHIKF outbreak. The laboratory diagnosis of CHIKF involves isolation and characterization of the virus, detection of viral nucleic acid and antibody detection ${ }^{5}$. Diagnosis of CHIKF using molecular techniques such as RT-PCR, RT-LAMP, qRT-PCR are the most widely acceptable due to their high specificity and sensitivity ${ }^{6}$. However, serological tests aimed at detecting serum IgM and/or IgG are the most popular methods of CHIKF diagnosis as they are the cheapest of all the diagnostic techniques and most convenient to conduct on patients ${ }^{7}$.

From the standpoint of epidemiology, Chikungunya fever (CHIKF) outbreaks tend to disappear for a sizable period of time from a particular part of the world before remerging again. For instance, the CHIKF outbreak around the Indian Ocean in 2005 have occurred nearly after three decades ${ }^{8}$. In Asia, the first outbreak was reported in Thailand in $1958^{9}$. Since then, the disease has been reported in many countries of Southeast Asia. India first reported the CHIKF outbreak in 1964 in its southern regions ${ }^{10}$. Being the country adjacent to India, Bangladesh documented the first outbreak of the disease in the year 2008 at Rajshahi and Chapainawabganj, the border-districts with India ${ }^{11}$. Since then, the disease has behaved as a re-emergent disease in the country with outbreaks occurring in 2011 at the sub-district of Dohar, Dhaka ${ }^{12}$ and again, in 2012 in the village of Palpapara of Tangail district ${ }^{13}$. 
Dhaka, the capital of Bangladesh, experienced the latest outbreak of CHIKF in the monsoon season of 2017. This epidemic was widely covered by the country's mainstream media ${ }^{14}$. A government organization, Institute of Epidemiology, Disease Control and Research (IEDCR), actively monitored the epidemic. Based on their newsletter on CHIKV, the epidemic peaked between early May and late July of 2017. Their newsletter also reported that 12060 out of 13814 people with CHIKV-related symptoms had visited three of the most prominent public hospitals along with IEDCR itself for clinical aid. Moreover, there was no alert of any other arbovirus-related diseases in the country at that time ${ }^{15}$. A few scientific reports studying this Dhaka epidemic of 2017 have already have been published 7,16,17. We believe findings of the current research will complement these previously published documents and add to the knowledge that already prevails on CHIKF seroepidemiology in Dhaka. Subsequently, identifying and controlling the risk factors of the infections might be much easier for health workers and policymakers to maintain a surveillance system against any CHIKF epidemics in the future.

\section{Materials and Methods}

\section{Study framework}

This cross-sectional epidemiological study was carried out in Dhaka, Bangladesh from July 17, 2017 to August 5, 2017. The study comprised individuals who were suffering from CHIKFrelated symptoms and came to the Ad-Din Hospital for a Chikungunya infection test. A total of 100 participants were selected based on a combination of inclusion and exclusion criteria. The inclusion criteria included those who were suffering from CHIKF-related symptoms such as high fever and joint pain accompanied by rash, severe headache. The exclusion criteria involved screening out individuals who had a history of suffering from dengue fever, arthralgia and arthritis. Each eligible respondent was interviewed personally after they were detailed on the process and purpose of the study and they provided their verbal consent. Since the patient came to the hospital for Chikungunya diagnosis, they already provided their blood to the hospital authority for the test. We did not have to physically puncture them for blood collection solely for our research, rather the blood specimen in our research was from the blood that was collected for the diagnosis purpose. A structured interview schedule was filled out based on the interview. This interview schedule consisted of two sections: the respondent's demographic data and data on the CHIKF-related signs and symptoms experienced. Afterwards, every respondent was assigned a unique identification number(ID), which was later used for serodiagnosis and data analysis.

\section{Sample collection and processing}

All 100 patients were detailed about the purpose and the process of the study. Blood specimens were collected, serum was separated and extracted as described by Mahbub et al. ${ }^{18}$. Three milliliters of blood were taken from each participant in a sterile glass test tube. The test tubes were allowed to rest at a slanted position for 1 hour for the blood to clot. Serum, collected at the upper level of the test tube, was transferred to a sterile Eppendorf tube with a Pasteur pipette. These tubes were centrifuged (Eppendorf, Germany; Model: 22331) at 3000 rpm for 10 minutes so that the remaining blood cells settled down. Clear serum was then transferred to another sterile Eppendorf tube and stored at $20^{\circ} \mathrm{C}$ until serodiagnosis.

\section{Serodiagnosis}

Every serum sample was tested qualitatively for the presence of anti-CHIKV IgG and/or IgM antibodies using a commercially available immunochromatographic assay kit called Standard Q Chikungunya IgM/IgG Test (Product Cat. No. 09CHI20D. Manufacturer: SD BIOSENSOR, Republic of Korea $)^{19}$. The conduction of the tests and the interpretation of the results were carried out strictly following the instruction manual provided with the kit.

\section{Processing and analysis of data}

Frequency distributions of the respondents based on their serostatus of anti-CHIKV antibodies, their clinical profiles and socio-demographic factors were made. Chi-square tests were employed as a measure of association. Subsequently, a multiple logistic regression model was set up choosing variables $(\mathrm{P}<0.25)$ from the Chi-square tests to avoid missing essential covariates in the model ${ }^{20}$. The discriminatory performance of the model was tested by using the "Receiver Operating Characteristic (ROC) Curve" and the area under the ROC curve was calculated ${ }^{21}$. A variable that yielded a $\mathrm{P}<0.05$ was considered statistically significant while one that emerged with a $\mathrm{P}<0.001$ was considered statistically highly significant. Data of the participants were tabulated and analyzed using SPSS version 25.0 (IBM Corp., Armonk, NY, USA). For the evaluation of the severity of pain, a 10-point numerical rating scale (NRS) (Table 4) was used $^{22}$. Ratings were categorized into mild (scores between 1 and 3), moderate (scores between 4 and 6), and severe (scores between 7 and 10). Follow-ups were made with the participants in intervals of 1 month for 3 months to check if they still experienced the pain in their joints.

\section{Results}

\section{Demographic characteristics of participants}

Of the 100 individuals who were randomly chosen and tested serologically for the presence of anti-CHIKV IgM and/or IgG, $74(74 \%, \mathrm{n}=100)$ individuals exhibited presence of IgM and/or IgG in their blood. This group was categorized as "seropositive," while the remaining 26 respondents were classified as seronegative. The $74 \%$ of the seropositive group was further broken down into three different classes: people having IgM only $(10 \%, \mathrm{n}=74)$, people having $\operatorname{IgG}$ only $(28 \%, \mathrm{n}=74)$, and people having both $\operatorname{IgG}$ and $\operatorname{IgM}(62 \%, n=74)$ (Figure 1$)$. 
Anti-CHIKV antibody distribution in seropositive group

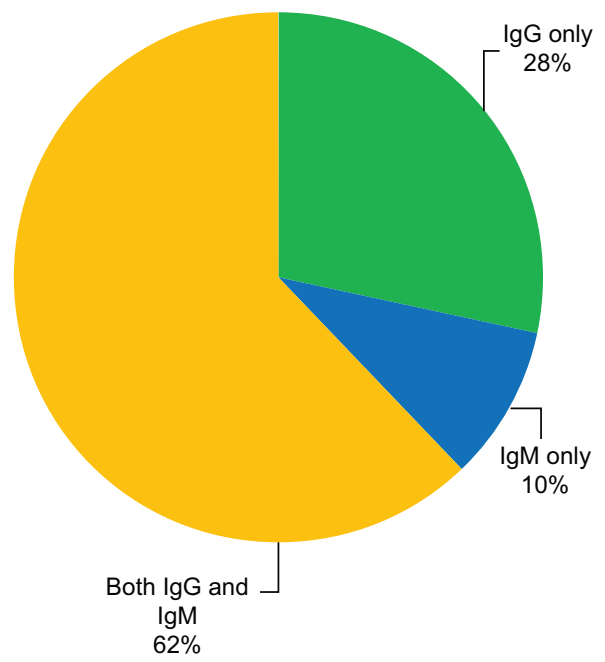

Fig.1. Classification of the seropositive group based on the presence of anti-CHIKV Immunoglobulins.

A look into the socio-demographic status of the studied patients (Table 1) depicted that $71 \%(\mathrm{n}=100)$ participants were male while the remaining $29 \%(\mathrm{n}=100)$ were female. The male to female ratio was calculated at 2.45:1. The mean age of the studied patients was $30.64(\mathrm{SD}=14.90)$. Children constituted $26 \%$ of the surveyed patients, while $69 \%$ were adults and $5 \%$ were elderly. Incidence rate was highest among adults aged between 18 and 59 years $(73 \%)$, followed by children $(63 \%)$ and elderly $(4 \%)$.

Table 1. Socio-demographic profile of the patients

\begin{tabular}{lccc}
\hline $\begin{array}{l}\text { Socio-demographic } \\
\text { characteristics }\end{array}$ & $\begin{array}{c}\text { Serostatus of anti-CHIKV antibodies } \\
\text { Seropositive } \\
(\%)\end{array}$ & $\begin{array}{c}\text { Total } \\
\text { Seronegative } \\
(\%)\end{array}$ & \\
\hline All & $74(100)$ & $26(100)$ & 100 \\
Age group (in years) & & & \\
Children $(<18)$ & $17(23.0)$ & $9(34.6)$ & 26 \\
Adults $(18-59)$ & $54(73.0)$ & $15(57.7)$ & 69 \\
Elderly $(>59)$ & $3(4.0)$ & $2(7.7)$ & 5 \\
Gender & & & \\
Male & $50(67.6)$ & $21(80.8)$ & 71 \\
Female & $24(32.4)$ & $5(19.2)$ & 29 \\
Marital Status & & & \\
Married & $36(48.6)$ & $5(19.2)$ & 41 \\
Unmarried & $38(51.4)$ & $21(80.8)$ & 59 \\
Education & & & \\
Uneducated & $5(6.8)$ & $0(0.0)$ & 5 \\
Primary & $8(10.8)$ & $2(7.7)$ & 10 \\
Secondary & $43(58.1)$ & $17(65.4)$ & 60 \\
Graduated & $18(24.3)$ & $7(26.9)$ & 25 \\
Occupation & & & \\
Service holder & $33(44.6)$ & $3(11.5)$ & 36 \\
Student & $35(47.3)$ & $20(76.9)$ & 55 \\
Housewife & $4(5.4)$ & $2(7.7)$ & 6 \\
Retired & $2(2.7)$ & $1(3.8)$ & 3 \\
\hline
\end{tabular}

Analysis of clinical and behavioral traits of the participants

The most prominent symptoms observed in the current study were high fever, polyarthralgia, rash, headache, myalgia, and vomiting
(Table 2). High fever $(\mathrm{P}=0.002)$ and $\operatorname{rash}(\mathrm{P}=0.008)$ demonstrated significant relationship with the presence of antiCHIKV IgM and/or IgG. In the multiple logistic regression analysis (Table 3), people with high fever $\left(\mathrm{e} 103^{0} \mathrm{~F}\right)$ were about 1.9 times (CI: $0.393-9.249$ ) more likely to be seropositive against CHIKV than that of people with fever below $103^{\circ} \mathrm{F}$. Likewise, someone with rash was about 1.8 times (CI: $0.525-$ 6.165) more likely to possess anti-CHIKV antibodies than that of the people having no rash. Meanwhile, the relationship between respondents having polyarthralgia and being immunopositive for anti-CHIKV IgM and/or IgG was highly significant at $\mathrm{P}<0.001$. In this case, the likelihood for someone with joint pain to be immunopositive for anti-CHIKV antibodies was about 11 times (CI: $1.792-67.042)$ higher than that of someone with no pain. Other clinical symptoms such as headache, vomiting, and myalgia did not show any significant relationship. In addition to the clinical manifestations, some behavioral attributes that could have contributed to the infection in the immunopositive group were

Table 2. Clinical profile and behavioral variables of the respondents in Dhaka CHIKF outbreak of 2017

\begin{tabular}{|c|c|c|c|}
\hline \multirow[t]{2}{*}{ Variables } & \multicolumn{2}{|c|}{ Serostatus of anti-CHIKV antibodies } & \multirow[t]{2}{*}{ P value } \\
\hline & $\begin{array}{c}\text { Seropositive } \\
(\%)\end{array}$ & $\begin{array}{c}\text { Seronegative } \\
(\%)\end{array}$ & \\
\hline$\overline{\text { All }}$ & $74(100)$ & $26(100)$ & \\
\hline \multicolumn{4}{|c|}{ Signs and Symptoms exhibited } \\
\hline \multicolumn{4}{|c|}{ High fever } \\
\hline$<103^{\circ} \mathrm{F}$ & $9(12.2)$ & $11(42.3)$ & $0.001 *$ \\
\hline$\geq 103^{\circ} \mathrm{F}$ & $65(87.8)$ & $15(57.7)$ & \\
\hline \multicolumn{4}{|c|}{ Polyarthralgia } \\
\hline No & $5(6.8)$ & $13(50.0)$ & $0.000 * *$ \\
\hline Yes & $69(93.2)$ & $13(50.0)$ & \\
\hline \multicolumn{4}{|l|}{ Rash } \\
\hline No & $29(39.2)$ & $18(69.2)$ & $0.008^{*}$ \\
\hline Yes & $45(60.8)$ & $8(30.8)$ & \\
\hline \multicolumn{4}{|l|}{ Headache } \\
\hline No & $30(40.5)$ & $11(42.3)$ & 0.875 \\
\hline Yes & $44(59.5)$ & $15(57.7)$ & \\
\hline \multicolumn{4}{|l|}{ Vomiting } \\
\hline No & $51(68.9)$ & $21(80.8)$ & 0.247 \\
\hline Yes & $23(31.1)$ & $5(19.2)$ & \\
\hline \multicolumn{4}{|l|}{ Myalgia } \\
\hline No & $27(36.5)$ & $11(42.3)$ & 0.599 \\
\hline Yes & $47(63.5)$ & $15(57.7)$ & \\
\hline \multicolumn{4}{|c|}{ Selected behavioural variables: } \\
\hline \multicolumn{4}{|c|}{ Travelled outside Dhaka before illness } \\
\hline No & $64(86.5)$ & $25(96.2)$ & 0.175 \\
\hline Yes & $10(13.5)$ & $1(3.8)$ & \\
\hline \multicolumn{4}{|c|}{ Use of mosquito control measures } \\
\hline No & $41(55.4)$ & $17(65.4)$ & 0.375 \\
\hline Yes & $33(44.6)$ & $9(34.6)$ & \\
\hline \multicolumn{4}{|c|}{ Household members with similar signs and symptoms } \\
\hline No & $34(45.9)$ & $20(76.9)$ & $0.006^{*}$ \\
\hline Yes & $40(54.1)$ & $6(23.1)$ & \\
\hline \multicolumn{4}{|c|}{ Availability of stagnant water near the residence } \\
\hline No & $0(0.0)$ & $0(0.0)$ & - \\
\hline Yes & $74(100.0)$ & $26(100.0)$ & \\
\hline
\end{tabular}


also investigated. About $46 \%(n=74)$ of the seropositive patients informed that they had at least one household member who exhibited similar CHIKF-related symptoms. From a statistical point of view, the relationship between a person immunopositive for anti-CHIKV antibodies and having at least one household member with similar ailments was significant at a P-value of 0.006 .

Table 4 depicts an in-depth look at the polyarthralgia profile of the seropositive group of the studied patients. Sixty-nine seropositive participants $(93.3 \%, \mathrm{n}=74)$ complained of pain in

Table 3. Results from Multiple Logistic Regression model various joints of their bodies during the duration that they were ill. To understand the intensity of pain they experienced, the study group was asked to rate the severity of their pain. Forty-two seropositive individuals $(56.8 \%, \mathrm{n}=74)$ rated their pain in the severe class. This was highest, followed by 28 individuals $(37.8 \%$, $\mathrm{n}=74$ ) whose pain was moderately severe. Seventy-two respondents $(97.3 \%, \mathrm{n}=74)$ informed that they had taken some form of medications to reduce the pain but to no avail.

The multiple logistic regression model was evaluated using the ROC curve. The area under the ROC curve (Figure 2) was 0.881

\begin{tabular}{lc}
\hline Variables & $\begin{array}{c}\text { Odds Ratio (OR) } \\
(95 \% \text { confidence interval) }\end{array}$ \\
\hline High Fever & 1 \\
$<103^{\circ} \mathrm{F}$ & $1.907 *(0.393-9.244)$ \\
$\geq 103^{\circ} \mathrm{F}$ & 1 \\
Polyarthralgia & $10.962 * *(1.792-67.042)$ \\
No & 1 \\
Yes & $1.799 *(0.525-6.165)$ \\
Rash & 1 \\
No & $1.530(0.394-5.938)$ \\
Yes & 1 \\
Vomiting & 1 \\
No & \\
Yes & $9.379(0.718-122.540)$ \\
Travelling outside Dhaka & 1 \\
No & \\
Yes & \\
Any other members of household with similar signs and symptoms & $5.905 *(1.592-21.906)$ \\
No & \\
Yes &
\end{tabular}

$* \mathrm{P}<0.05$. **P $<0.001$

Table 4. In-depth investigation of the arthralgia profile of the respondents

\begin{tabular}{lcc}
\hline Variables & \multicolumn{1}{c}{ Serostatus of anti-CHIKV antibodies } & Seronegative $(\%)$ \\
\cline { 2 - 3 } & Seropositive $(\%)$ & $13(50.0)$ \\
All $74(100)$ & $26(100)$ & $5(19.2)$ \\
Duration of pain & $5(6.8)$ & $4(15.4)$ \\
$\quad$ No arthralgia & $13(17.6)$ & $4(15.4)$ \\
$\quad$ Less than 7 days & $10(13.5)$ & $0(0.0)$ \\
$\quad$ Acute (7-14 days) & $41(55.4)$ & $5(19.2)$ \\
Subacute (up to 90 days) & $5(6.8)$ & $3(11.5)$ \\
Chronic (more than 90 days) & $2(2.7)$ & $14(53.8)$ \\
Pain intensity & $2(2.7)$ & $4(15.4)$ \\
$\quad$ No pain & $28(37.8)$ & \\
$\quad$ Mild (NRS scores of $1-3)$ & $42(56.8)$ & \\
$\quad$ Moderate (NRS scores of $4-6)$ & & \\
$\quad$ Severe (NRS scores of $7-10)$ & $7(26.92)$ & \\
Analgesics used & $19(73.08)$ & \\
No 2 (2.70) & & \\
Yes 72 (97.30) & & \\
\hline
\end{tabular}


(CI: $0.807-0.956)$, depicting a "good" Discriminatory Performance (DP) of the model ${ }^{21}$.

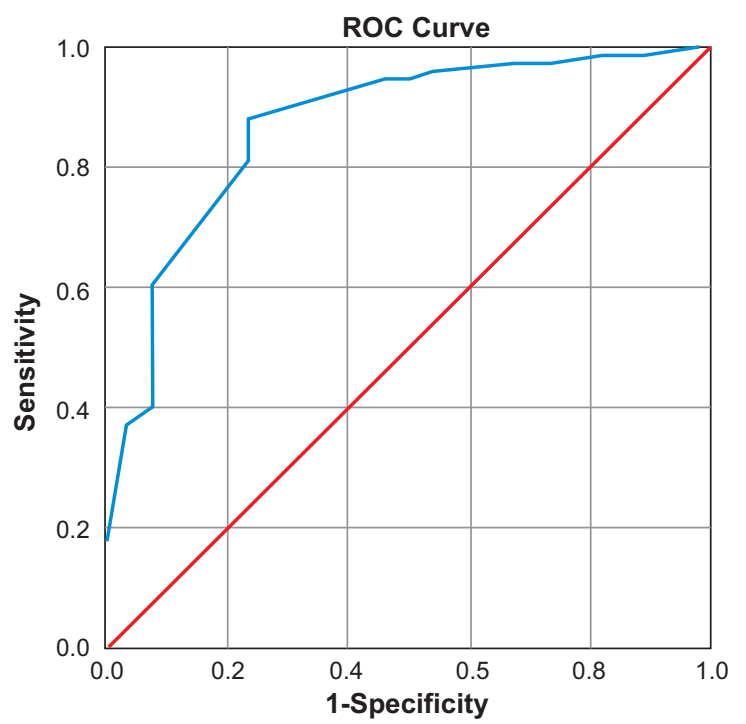

Fig. 2. ROC curve for the Logistic regression model showing an area of 0.881 (CI: 0.807 - 0.956).

\section{Discussion}

Dhaka is a city with a population density of 23,234 people per square kilometer ${ }^{23}$. The climate of the city is generally hot, wet, and humid, with distinct monsoon periods. About $87 \%$ of the annual average rainfall of 2,123 $\mathrm{mm}$ occurs between May and October $^{24}$. Over one-third of the population is poor and lives in densely populated slumps ${ }^{25}$. During the monsoon, water bodies scattered throughout the city rise in levels. Garbage, such as plastic containers and rubber tires, holds clear water, providing suitable growth sites for mosquito larvae ${ }^{26}$. On top of that, Dhaka witnessed an alarming level of rainfall in the year 2017 during the CHIKF epidemic. Both the CHIKF epidemic and the high rainfall were covered by the country's news media ${ }^{9,19}$. Also, as calculated by Dhar-Chowdhury et al. ${ }^{28}$, the Breteau Index (BI or number of $A$. aegypti infested containers per 100 houses inspected) in Dhaka was as high as in between the range of 52.0 and 63.4. Therefore, it is not surprising that the CHIKV vector is so widespread in most of the city's administrative units.

The overall seroprevalence of anti-CHIKV IgM and/or IgG in this study was $74 \%(n=100)$. Out of these, $10 \%(n=74)$ had IgM only while $62 \%$ had both IgM and IgG, indicating recent infections in the individuals. On the other hand, $28 \%$ of the immunopositive group $(n=74)$ had only IgG in their serum indicating past infections ${ }^{29}$. The overall seroprevalence of $74 \%$ was in close agreement to that reported by Rashid et al. ${ }^{7}$ who investigated the same outbreak.

The investigated patients of the present study exhibited the typical symptoms of CHIKV infection, including fever, polyarthralgia, rash, headache, vomiting, myalgia. Like most reports ${ }^{30}, 59.5 \%$ $(n=74)$ of the seropositive patients reported that the illness began with sudden onset of high fever followed by debilitating joint pain. In contrast, Hossain et al. ${ }^{16}$ and Anwar et al. ${ }^{17}$ who conducted their own separate investigations of the same outbreak, reported that the major proportion of the patients in their study experienced pain in joints as the initial symptom. High fever and arthralgia together were documented in $93.2 \%(n=74)$ of the seropositive respondents confirmed with anti-CHIKV antibody. Fever and arthralgia can therefore be considered the hallmark of our study. This finding also conforms with previous epidemics in the Réunion Island ${ }^{30-32}$, Panama ${ }^{33}$, Suriname ${ }^{34}$, Singapore ${ }^{35}$ and Dhaka ${ }^{17}$. Four participants $(5.41 \%, \mathrm{n}=74)$ with anti-CHIKV antibodies reported to have experienced no arthralgia but having th-e other typical symptoms of CHIKF. A similar finding has also been reported by Osterrieth et al. ${ }^{36}$.

About $97.3 \%$ of the studied individuals suffered from high fever $(\mathrm{P}=0.001$; Table 2$)$ at an average highest body temperature of $103.6^{\circ} \mathrm{F}(\mathrm{SD}=1.30)$ that lasted for 4.86 days $(\mathrm{SD}=2.95)$. Hossain et al. ${ }^{16}$ reported this very same finding in their study. Remarkably, $5.4 \%(n=74)$ of the seropositive group informed that their body temperature spiked as high as $106^{\circ} \mathrm{F}$, whereas another $10.8 \%$ said theirs reached $105^{\circ} \mathrm{F}$. Furthermore, the multiple logistic regression model (Table 3) demonstrated that participants recording the highest body temperature greater than or equal to $103^{\circ} \mathrm{F}$ were at 1.9 times higher odds of being seropositive for anti-CHIKV antibody.

Polyarthralgia was most deeply investigated because of the adverse impact it had on the quality of daily life of the patients. From a statistical standpoint, the relationship between seropositivity for anti-CHIKV antibody and suffering from arthralgia was highly significant at $\mathrm{P}<0.001$ (Table 2). In the multiple logistic regression analysis (Table 3 ), a person with joint pain was about 11 times more likely to be positive for anti-CHIKV $\operatorname{IgM}$ and/or IgG than a person who did not have pain in the joints. The patients were further classified (Table 4) based on the duration for which they suffered. Those who were immunopositive for anti-CHIKV antibodies, $41(55.4 \%, \mathrm{n}=74)$ suffered for durations ranging from 15 to 89 days, while 5 participants $(6.8 \%, \mathrm{n}=74)$ had the pain in the chronic stage with the duration of pain exceeding 90 days. As it is common that arthralgia associated with CHIKV infection has little or no response to analgesics ${ }^{37}$, we inquired every participant if they took any medication for the pain. Locally available pain medicines were used, but not one of the respondents had any relief from the pain. Contrarily, they complained about hindered daily life; some patients even informed this pain was the worst they had ever suffered from. This same complaint was registered by subjects of the epidemics in Madras City ${ }^{38}$ and Réunion Island ${ }^{31}$. As a result, we tried to evaluate the severity of pain experienced by our respondents based on a 10 - point numerical rating scale (NRS) (Table 4). Forty-two $(56.8 \%, n=74)$ of the seropositive respondents fell in the category of severe joint pain (NRS score 
$7-10)$, and $28(37.8 \%)$ recorded moderate pain intensity (NRS score $4-6)$. As corroborated by our study, the overall measurement of pain intensity was also generally high in other similar seroepidemiology of CHIKV ${ }^{39}$, especially those conducted in Dhaka in $2017^{16,17}$. The reason behind prolonged suffering from pain in the joints is still poorly understood. Studies have speculated that the persistence of this particular symptom of CHIKF could be due to the inflammatory response of the host, the presence of viral products in joint tissues and macrophages for long periods of time and the involvement of some autoimmune responses in pathogenesis ${ }^{37}$.

Apart from fever and arthralgia, severe headaches, skin rash, and vomiting were other notable symptoms observed. Among these, rash development had statistical significance with a P-value of 0.008 (Table 2). The logistic regression model gave a person with rash on their skin 1.8 times (Table 3 ) more chance of being immunopositive for anti-CHIKV antibodies.

As mentioned earlier ${ }^{23-26,28}$, Dhaka provides a favorable breeding ground for CHIKV vectors. In addition, the year 2017 produced atypically high rainfall. We tried to see if this aspect had any effect on the outbreak of CHIKF. Respondents were asked if they had any source of stagnant water surfaces around their area of residence. This could have been anything from a puddle of clean water or a potted plant at home. As Dhaka is littered with waterlogged streets during seasons of high rainfall, all 100 of the participants in our research informed that they had some form of stagnant waterbodies near them. Although this was expected, it could not be analyzed with a statistical tool (Table 2) due to the lack of variability in data.

Another interesting aspect that caught our attention was that $54.1 \%$ of the seropositive individuals in our target group noticed similar CHIKF symptoms in other members of their household. People who claimed to have witnessed other members of their household exhibiting CHIKF-like symptoms were at greater odds to be seropositive for anti-CHIKV antibodies ( $\mathrm{P}=0.006$; O.R: 5.905, CI: 1.592-21.906). Although the other members of the households could not be included in the study, this finding, however appeared to be coherent with the behavioral pattern of Aedes vectors. The vector is known to live in close proximity to people and bite several people at a time for the same blood meal ${ }^{40}$. They also have a flight range of merely a hundred meters ${ }^{41}$. These characteristics of $A$. aegypti could point to the fact that people living in the same household with symptoms of CHIKF are prone to be bitten by the same infected mosquito so that they succumb to the disease at the same time.

The clinical profile demonstrated by the participants of this study conformed with several previously documented CHIKF outbreaks worldwide. However, this study is not free from limitations. In comparison to other contemporary reports, the sample size in our study was smaller. This was due to the fact that a great deal of effort was required to explain the concept of the study to people and then convince them to participate. Moreover, patients in this study were recruited through a hospital and according to Hossain et al. the number of hospital confirmed cases were low because of the high expense of tests and scarcity of diagnostic facilities ${ }^{16}$. The Directorate General of Health Services (DGHS, the health services regulatory authority in Bangladesh) actively discouraged suspected patients from laboratory diagnosis. The rationales of the DGHS behind this was that significant proportion of people in risk were of the low-income group of the society and that the fatality rate of this infection is extremely low ${ }^{16,17}$.

With no licensed vaccines and drugs against the virus itself ${ }^{42}$, the disease can only be mitigated by controlling the Aedes vectors. Bangladesh, in that regard, is at risks as mosquito-borne diseases are relatively common. In order to prevent future epidemics, robust surveillance and mosquito-control systems need to be implemented. At the same time, research should be directed towards the genome of the Chikungunya virus to monitor its mutations and possible effects.

\section{Declarations}

\section{Acknowledgements}

We are grateful to the participants of this study and Mr. Md. Touhidul Alam, Assistant Professor, Dhaka School of Economics, the University of Dhaka, for his contributions in data analyses. We are also grateful to the laboratory staff of Ad-Din Hospital, Dhaka for their help and very kind cooperation.

\section{Competing Interests}

None of the authors have any conflict of interest to declare.

\section{Funding}

Not Applicable.

\section{References}

1. Word Health Organization. Guidelines on Clinical Management of Chikungunya Fever. Published online 2008:18.

2. Schuffenecker I, Iteman I, Michault A, et al. Genome microevolution of chikungunya viruses causing the Indian Ocean outbreak. PLoS Med. 2006;3(7):e263-e263. doi:10.1371/journal.pmed.0030263.

3. Sharif N, Sarkar MK, Ferdous RN, et al. Molecular Epidemiology, Evolution and Reemergence of Chikungunya Virus in South Asia. Front Microbiol. 2021;12(June):1-14. doi:10.3389/fmicb.2021.689979.

4. Cabral-Castro MJ, Cavalcanti MG, Peralta RHS and Peralta JM. Molecular and serological techniques to detect co-circulation of DENV, ZIKV and CHIKV in suspected dengue-like syndrome patients. J Clin Virol. 2016;82:108-111. doi:10.1016/J.JCV.2016.07.017

5. Chikungunya virus CDC. Accessed November 29, 2021. https:// www.cdc.gov/chikungunya/index.html

6. Calvo EP, Sánchez-Quete F, Durán S, Sandoval I and Castellanos JE. Easy and inexpensive molecular detection of dengue, chikungunya and zika viruses in febrile patients. Acta Trop. 2016;163:32-37. doi:10.1016/ J.ACTATROPICA.2016.07.021

7. Mdho R, MH P, AI, et al. Seroprevalence of Chikungunya during Outbreak in Dhaka, Bangladesh in 2017. J Virol Antivir Res. 2018;07(02):2-5. doi:10.4172/2324-8955.1000184

8. Ravi V. Re-emergence of chikungunya virus in India. Indian J Med Microbiol. 2006;24(2):83-84. doi:10.4103/0255-0857.25175 
9. Hammon WM, Rundnick A and Sather GE. Viruses associated with epidemic hemorrhagic fevers of the Philippines and Thailand. Science. 1960;131(3407):1102-1103.

10. Kalantri SP, Joshi R and Riley LW. Chikungunya epidemic: An Indian perspective. Natl Med J India. 2006;19(6):315-322.

11. ICDDR. First identified outbreak of Chikungunya in Bangladesh , 2008. Heal Sci Bull. 2009;7(1):1-6.

12. Khatun $\mathrm{S}$, Chakraborty A, Rahman M, et al. An outbreak of chikungunya in rural Bangladesh, 2011. PLoS Negl Trop Dis. 2015;9(7):1-9. doi:10.1371/journal.pntd.0003907

13. Salje H, Lessler J, Paul KK, et al. How social structures, space, and behaviors shape the spread of infectious diseases using chikungunya as a case study. Proc Natl Acad Sci. 2016;113(47):13420-13425. doi:10.1073/ pnas. 1611391113

14. Chikungunya onslaught on | The Daily Star. Accessed July 26, 2018. https:/ /www.thedailystar.net/backpage/chikungunya-onslaught-1432504

15. Chikungunya News Letter. Accessed March 13, 2020. https:// www.iedcr.gov.bd/index.php/pheoc/chikungunya-newsletter

16. Hossain MS, Hasan MM, Islam MS, et al. Chikungunya outbreak (2017) in Bangladesh: Clinical profile, economic impact and quality of life during the acute phase of the disease. PLoS Negl Trop Dis. 2018;12(6). doi:10.1371/journal.pntd.0006561

17. Anwar S, Mourosi JT, Khan MF, Ullah MO, Vanakker OM and Hosen MJ. Chikungunya outbreak in Bangladesh (2017): Clinical and hematological findings. PLoS Negl Trop Dis. 2020;14(2). doi:10.1371/ journal. pntd.0007466

18. Mahbub MM, Ahsan CR, Yasmin M and Nessa J. Analysis of Different Prognostic Indicators for Malnutrition and Shigella flexneri Infection Among the Children in Bangladesh. Indian J Microbiol. 2012;52(3):400405. doi:10.1007/s12088-012-0247-1

19. Products - STANDARD Q Chikungunya IgM/IgG. Accessed November 3, 2020. http://sdbiosensor.com/xe/product/4091? category $=2306$

20. Hosmer DW and Lemeshow S. Applied Logistic Regression. Wiley Ser Probab Sattistics. 2000;(1):373. doi:10.2307/2074954

21. Kleinbaum DG, Klein M and Pryor ER. Logistic regression: a self-learning text. 2002

22. Jones KR, Vojir CP, Hutt E and Fink R. Determining mild, moderate, and severe pain equivalency across pain- intensity tools in nursing home residents 1. 2007;44(2):305-314. doi:10.1682/JRRD.2006.05.0051

23. Dhaka Population 2018 (Demographics, Maps, Graphs). Accessed July 26, 2018. http://worldpopulationreview.com/world-cities/dhakapopulation/

24. Dhaka, Bangladesh Weather Averages | Monthly Average High and Low Temperature | Average Precipitation and Rainfall days | World Weather Online. Accessed December 31, 2018. https://www. world weatheronline. com/dhaka-weather-averages/bd.aspx

25. Islam MA, Ahmed MU, Begum N, et al. Molecular Characterization and Clinical Evaluation of Dengue Outbreak in 2002 in Bangladesh. 2006. 7:85-91.

26. Hashizume M, Dewan AM, Sunahara T, Rahman MZ and Yamamoto T. Hydroclimatological variability and dengue transmission in Dhaka, Bangladesh/ : a time-series study. Published online 2012.
27. Rain causes waterlogging, traffic chaos in Dhaka| Dhaka Tribune. Accessed January 3, 2019. https://www.dhakatribune.com/bangladesh/ nation/2017/08/03/rain-causes-waterlogging-traffic-chaos-dhaka

28. Dhar-Chowdhury P, Haque CE, Lindsay R and Hossain S. Socioeconomic and ecological factors influencing aedes aegypti prevalence, abundance, and distribution in Dhaka, Bangladesh. Am J Trop Med Hyg. 2016;94(6):1223-1233. doi:10.4269/ajtmh.15-0639

29. Biswas B, Amin S, Azad MAK, et al. A study on the dangerous outbreak of chikungunya in Chittagong, including a limited survey around that city of Bangladesh. Int J Community Med Public Heal. 2019;6(11):4677. doi:10.18203/2394-6040.ijcmph20195039

30. de Valk H, Balleydier E, Solet J-L, et al. A Major Epidemic of Chikungunya Virus Infection on Réunion Island, France, 2005-2006. Am J Trop Med Hyg. 2018;77(4):727-731. doi:10.4269/ajtmh.2007.77.727

31. Borgherini G, Poubeau P, Staikowsky F, et al. Outbreak of Chikungunya on Reunion Island: Early Clinical and Laboratory Features in 157 Adult Patients. Clin Infect Dis. 2007;44(11):1401-1407. doi:10.1086/517537

32. Thiberville S-D, Boisson V, Gaudart J, Simon F, Flahault A and de Lamballerie X. Chikungunya Fever: A Clinical and Virological Investigation of Outpatients on Reunion Island, South-West Indian Ocean. PLoS Negl Trop Dis. 2013;7(1):e2004. https://doi.org/10.1371/journal.pntd.0002004

33. Carrera JP, Díaz Y, Denis B, et al. Unusual pattern of chikungunya virus epidemic in the Americas, the Panamanian experience. PLoS Negl Trop Dis. 2017;11(2):1-23. doi:10.1371/journal.pntd.0005338

34. van Genderen FT, Krishnadath I, Sno R, Grunberg MG, Zijlmans W and Adhin MR. First Chikungunya Outbreak in Suriname; Clinical and Epidemiological Features. PLoS Negl Trop Dis. 2016;10(4):e0004625e0004625. doi:10.1371/journal.pntd.0004625

35. Win MK, Chow A, Dimatatac F, Go CJ and Leo YS. Chikungunya fever in Singapore: Acute clinical and laboratory features, and factors associated with persistent arthralgia. J Clin Virol. 2010;49(2):111-114. doi:10.1016/ j.jev.2010.07.004

36. OSTERRIETH P, BLANES-RIDAURA G. [Research on the Chikungunya virus in the Belgian Congo. I. Isolation of the virus in upper Uele]. Ann la Soc belge Med Trop. 1960. 40:199-203.

37. de Brito CAA, von Sohsten AKA, de Sá Leitão CC, et al. Pharmacologic management of pain in patients with Chikungunya: A guideline. Rev Soc Bras Med Trop. 2016;49(6):668-679. doi:10.1590/0037-8682-0279-2016

38. De Ranitz CM, Myers RM, Varkey MJ, Isaac ZH and Carey DE. Clinical impressions of chikungunya in Vellore gained from study of adult patients. Indian J Med Res. 1965;53(8):756.

39. Sissoko D, Malvy D, Ezzedine K, et al. Post-epidemic Chikungunya disease on Reunion Island: course of rheumatic manifestations and associated factors over a 15-month period. PLoS Negl Trop Dis. 2009;3(3).

40. Gibbons R V and Vaughn DW. Dengue: An Escalating Problem Clinical review Dengue: an escalating problem. Source BMJ Br Med J. 2002;324113176(7353):1563-1566. doi:10.1136/bmj.324.7353.1563

41. Harrington LC, Scott TW, Lerdthusnee K, et al. Dispersal of the dengue vector Aedes aegypti within and between rural communities. Am J Trop Med Hyg. 2005;72(2):209-220. doi:72/2/209 [pii]

42. Thiberville SD, Moyen N, Dupuis-Maguiraga L, et al. Chikungunya fever: Epidemiology, clinical syndrome, pathogenesis and therapy. Antiviral Res. 2013;99(3):345-370. doi:10.1016/J.ANTIVIRAL.2013.06.009 